\title{
Diagnostic work-up strategy for periprosthetic joint infections after total hip and knee arthroplasty: a 12-year experience on 320 consecutive cases
}

Dirk Zajonz ${ }^{1 * \dagger}$, Lena Wuthe ${ }^{1 \dagger}$, Solveig Tiepolt², Philipp Brandmeier ${ }^{3}$, Torsten Prietzel' ${ }^{1}$, Georg Freiherr von Salis-Soglio ${ }^{1}$, Andreas Roth ${ }^{1}$, Christoph Josten ${ }^{1}$, Christoph-E. Heyde ${ }^{1}$ and Mohamed Ghanem ${ }^{1}$

\begin{abstract}
Background: Elective knee and hip arthroplasty is followed by infections in currently about $0.5-2.0 \%$ of cases - a figure which is on the increase due to the rise in primary implants. Correct diagnosis early on is essential so that appropriate therapy can be administered. This work presents a retrospective analysis of the diagnoses of patients suffering infections after total hip or knee arthroplasty.

Methods: 320 patients with prosthetic joint infection (PJI) following knee or hip arthroplasty were identified. They comprised a) 172 patients with an infection after total hip arthroplasty (THA): $56 \%$ females $(n=96)$ and $44 \%$ males $(n=76)$ with a mean age of 70.9 (39-92) years; and 148 patients with an infection after total knee arthroplasty (TKA): $55 \%$ females $(n=82)$ and $45 \%$ males $(n=66)$ with a mean age of $70.7(15-87)$ years.

Results: Although significantly more TKA than THA patients reported pain, erythema, a burning sensation and swelling, no differences between the two groups were observed with respect to dysfunction, fever or fatigue. However, significant differences were noted in the diagnosis of loosening (THA $55 \%$, TKA $31 \%, p<0.001$ ) and suspected infection using conventional X-rays (THA $61 \%$, TKA $29 \%, p<0.001$ ). FDG-PET-CT produced very good results in nearly $95 \%$ of cases. There were no differences between THA and TKA patients regarding levels of inflammation markers. Histological evaluation proved to be significantly better than microbiological analysis.

Summary: The clinical picture may be non-specific and not show typical inflammatory symptoms for a long time, particularly in PJI of the hip. As imaging only provides reliable conclusions after the symptoms have persisted for a long time, morphological imaging is not suitable for the detection of early infections. FDG-PT-CT proved to be the most successful technique and is likely to be used more frequently in future. Nevertheless, there are currently no laboratory parameters which are suitable for the reliable primary diagnosis of PJI. Diagnosis requires arthrocentesis, and the fluid obtained should always be examined both microbiologically and histologically.
\end{abstract}

Keywords: Prosthetic joint infection, Diagnosis, THA, TKA

\footnotetext{
* Correspondence: Dirk.Zajonz@medizin.uni-leipzig.de

${ }^{\dagger}$ Equal contributors

'Department of Orthopaedic Surgery, Traumatology and Plastic Surgery,

University Hospital Leipzig, Liebigstrasse 20, 04103 Leipzig, Germany
}

Full list of author information is available at the end of the article 


\section{Introduction}

Hip and knee replacements are among the most common operations worldwide. The quantity performed is increasing significantly in response to demographic change. For example, the number of total hip arthroplasties (THA) carried out in the US rose by a factor of 2.5 from 200,216 in 1993 to 497,419 in 2005. In the same period, the amount of primary total knee arthroplasties (TKA) grew 1.7-fold from 135,992 to 237,645 $[1,2]$. High expectations are attached to arthroplasty, which has been described as the operation of the century [3] due to its high success rate. Although the risk of complications (as with any medical procedure) cannot be excluded, the implantation of endoprostheses leads to problems in fewer than $10 \%$ of cases [4]. The main drawback occurring is aseptic loosening [4]. Although infections occur more rarely, they are one of the most feared complications and are potentially fatal. Reflecting the quantity of primary joint replacements, the number of infections diagnosed in the US has steadily increased: between 1990 and 2004, the number of hip arthroplasty infections more than tripled while knee arthroplasty infections increased almost six-fold [5].

Thanks to the introduction of perioperative antibiotic prophylaxis and the optimization of operating hygiene (such as the use of cleanroom standards in the operating theatre), infection rates following elective knee and hip arthroplasty have been reduced from about $10 \%$ in the 1960 s to currently about $0.5-2.0 \%[4,6-8]$. However, this rate climbs to about $5 \%$ after revision surgery, and to as high as $15-40 \%$ after reimplantations $[4,9,10]$. Infection creates a high psychological, physiological and financial burden for the patient, their family members, the attending physicians and cost carriers. Even if treatment is successful in a single operation, the antibiotic additives alone may cost as much as $€ 5000$ [11]. For complex revision surgery, Barrack et al. reported treatment costs exceeding \$50,000 back in 1999 [12].

Appropriate therapy depends on early, correct diagnosis. Given the usually enormous consequences often entailing explantation, diagnosis has to be as reliable as possible [13]. Since the clinical presentation of PJI varies greatly, a detailed medical history should be compiled addressing any existing risk factors [14]. In particular, the general clinical symptoms of inflammation may be non-specific in acute systemic or subacute local inflammation [14]. At any rate, pain in the replaced joint accompanied by fever needs to be diagnosed [9].

The aim of this study was to analyse retrospectively the diagnosis of patients with PJI in an attempt to identify any valid diagnostic parameters. Another goal was to identify any differences in the individual diagnostic factors concerning knee and hip arthroplasty infections.

\section{Material and methods}

Patients were considered to suffer infection if clinical and paraclinical examination methods resulted in a positive microbiological culture or positive histological findings in arthrocentesis or surgery specimens indicating prosthetic joint infection (PJI).

To select the patient cohort, all patients with the ICD-10 diagnosis code T84.5 (infection and inflammatory reaction due to internal joint prosthesis) who had been treated in our department between 1 January 2001 and 31 December 2012 were retrospectively identified. Patient data were gathered from archived medical records and electronic records in IS-H (SAP) (Siemens AG Healthcare Sector, Erlangen, Germany) as well as from radiological findings and images in SIENET MagicWeb/ACOM (Siemens AG Healthcare Sector, Erlangen, Germany). From this patient population, a total of 320 patients with PJI after TKA or THA were identified in whom an infection of knee or hip arthroplasty was first diagnosed in the above-mentioned period in our department.

All in all, 172 patients with periprosthetic infection after THA were identified. Comprising $56 \%$ (96) females and $44 \%$ (76) males, their mean age when infection was diagnosed was about 70.9 (39-92) years. Early infections (within the first six weeks after THA) accounted for $32 \%$ (55 patients), delayed infections (between six weeks and two years after THA) accounted for $27 \%$ (47) of infections, while in $38 \%$ (66), infection occurred over two years after THA. In four patients, the time of THA was unknown [7].

There were 148 cases of periprosthetic infection following TKA. The patient cohort consisted of $55 \%$ (82) females and $45 \%$ (66) males with a mean age of 70.7 (15-87) years. Early infection occurred in $20 \%$ (29 patients), delayed infection affected $42 \%$ (62), and in $36 \%$ (54) infection only occurred after at least two years. For three patients, the exact period since surgery was unknown [7].

General clinical symptoms like pain, fatigue and fever as well as clinical findings such as erythema, burning sensation, swelling, dysfunction and fistula formation were evaluated. Furthermore, the presence of sepsis was ascertained. The inflammation parameters CRP $(\mathrm{mg} / \mathrm{l})$, leukocytes $(\mathrm{Gpt} / \mathrm{l}), \mathrm{Hb}(\mathrm{mmol} / \mathrm{l})$ and sometimes PCT $(\mathrm{ng} / \mathrm{ml})$ were measured on admission or first presentation. Radiologists' findings using conventional $\mathrm{X}$-rays documenting described loosening or the suspicion of infection were taken into account. Suspected infections following analysis of CT, MRI, three-phase bone scintigraphy, anti-granulocyte scintigraphy or PET-CT (FDG-PET) by specialists were also included. Furthermore, the findings of microbiological and histological analyses of diagnostic arthrocentesis were compared to the definitive results during surgery. 
Statistical analysis was performed using the spreadsheet software Microsoft Excel (Microsoft Corporation, Redmond, USA). The $t$-test for two dependent samples was used to calculate significance, the level of significance being set at $5 \%(\alpha=0.05)$.

\section{Results}

\section{Clinical diagnosis}

The individual clinical symptoms are shown in Fig. 1. The high percentage of patients with THA or TKA infection suffering pain (TKA $95 \%$ (141), THA $89 \%$ (153)) and dysfunction in the joint concerned (THA 74 \% (128), TKA $85 \%$ (126)) was striking. Significantly more patients with TKA treatment reported pain than THA patients $(p=0.039)$. Furthermore, significant differences regarding erythema, burning sensation and swelling were reported by a higher proportion of patients with TKA infection (Fig. 1). There were no differences in the predominance of fever, fatigue or sepsis. Fistula formation occurred significantly more often following THA than TKA infection (THA $31 \%$ (54), TKA $19 \%$ (28); $\mathrm{p}=0.0067$ ).

\section{Laboratory diagnosis}

Data for CRP, leucocyte count and haemoglobin were available in 168 cases of THA infection. In 14 cases, the procalcitonin level had also been measured. Laboratory data was available for 145 cases of TKA infection, including in 13 cases the level of procalcitonin.

Mean CRP in patients with THA infection was about $98.2 \mathrm{mg} / \mathrm{l}(\mathrm{min} / \mathrm{max} \quad 0.89-438 \mathrm{mg} / \mathrm{l})$ and $\mathrm{mg} / \mathrm{l} 117.5$ $(1.12-515 \mathrm{mg} / \mathrm{l})$ in patients with TKA infection. The mean leukocyte count was $9.67 \mathrm{Gpt} / \mathrm{l}(3.4-30.8 \mathrm{Gpt} / \mathrm{l})$ in patients with THA infection and $10.57 \mathrm{Gpt} / \mathrm{l}(0.6-44 \mathrm{Gpt} / \mathrm{l})$ in patients with TKA infection. Mean PCT was $2.94 \mathrm{ng} / \mathrm{ml}$ $(0.07-15.16 \mathrm{ng} / \mathrm{ml})$ in patients with THA and $11.08 \mathrm{ng} / \mathrm{ml}$ $(0.03-100.3 \mathrm{ng} / \mathrm{ml})$ in patients with TKA infection. Mean $\mathrm{Hb}$ was $6.93 \mathrm{mmol} / \mathrm{l}(3.2-9.2 \mathrm{mmol} / \mathrm{l})$ in patients with TKA infection and $7.17 \mathrm{mmol} / \mathrm{l}(4.6-10.7 \mathrm{mmol} / \mathrm{l})$ in patients with THA infection. The differences between patients with THA and TKA infections were not significant.

The individual laboratory readings are compared between THA and TKA infections in Table 1, which also lists the numbers of patients with abnormal laboratory data.

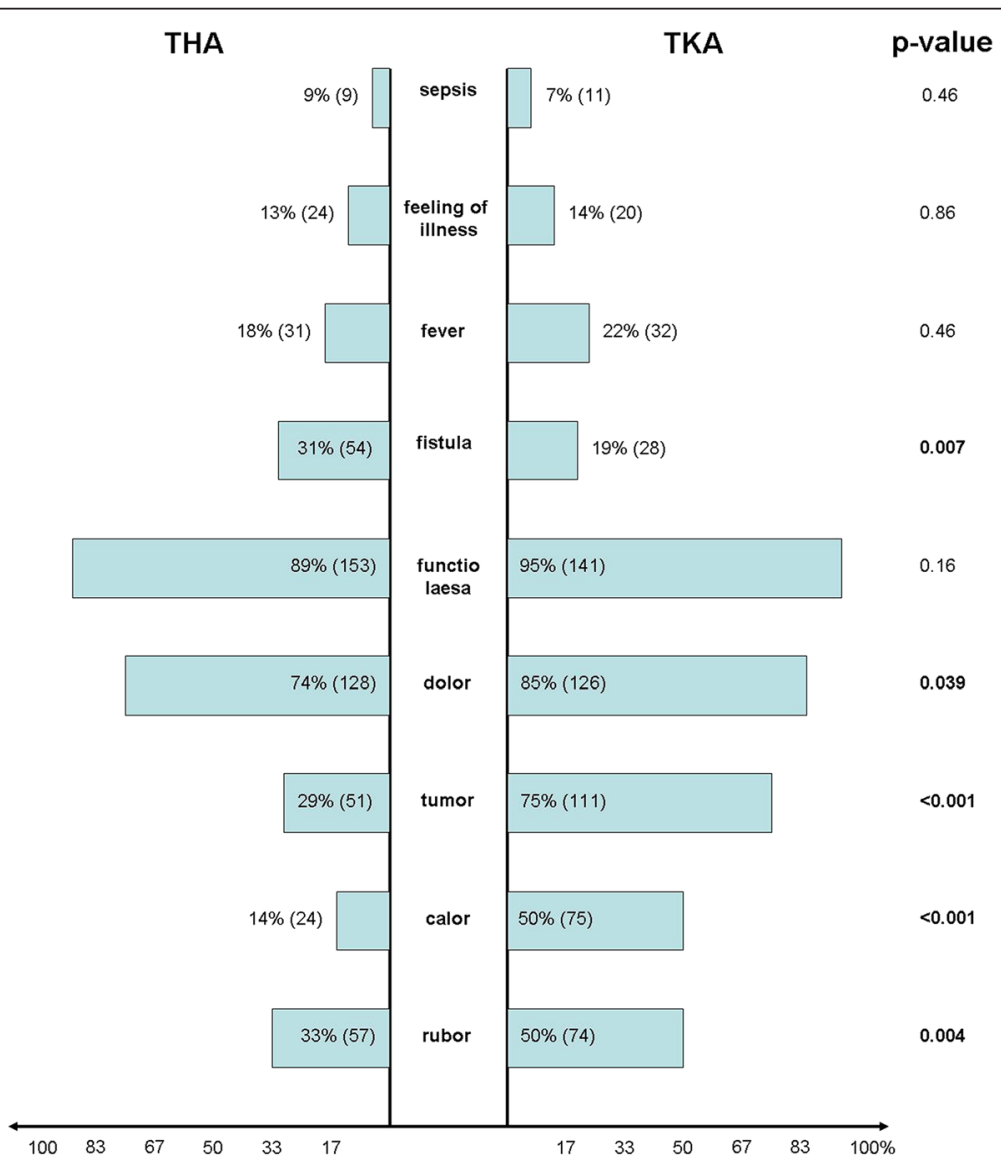

Fig. 1 Clinical symptoms. Graph showing percentage and absolute difference in clinical symptoms between THA (left) and TKA (right) infection as well as the p-value (statistical significances printed in bold type) 
Table 1 Laboratory readings. Shows the laboratory readings compared between THA and TKA infections and also the numbers of patients with abnormal laboratory data

\begin{tabular}{llllll}
\hline & $\begin{array}{l}\text { Percentage of positive } \\
\text { patients (absolutely) }\end{array}$ & $\begin{array}{l}\text { Arithmetic average } \\
\text { (min-max) }\end{array}$ & Standard & $\begin{array}{l}\text { Arithmetic average } \\
\text { (min-max) }\end{array}$ & $\begin{array}{l}\text { Percentage of positive } \\
\text { patients (absolutely) }\end{array}$ \\
\hline CRP & $95 \%(159)$ & $98.2(0.89-438)$ & $<5 \mathrm{mg} / \mathrm{l}$ & $117.5(1.12-515)$ & $93 \%(135)$ \\
Leukocytes & $39 \%(65)$ & $9.67(3.4-30.8)$ & $4-9 \mathrm{Gpt} / \mathrm{l}$ & $10.57(0.6-44)$ & $43 \%(63)$ \\
Procalcitonin (PCT) & $57 \%(8)$ & $2.94(0.07-15.16)$ & $<0.5 \mathrm{ng} / \mathrm{ml}$ & $11.08(0.03-100.3)$ & $54 \%(7)$ \\
Hemoglobin level & $79 \%(133)$ & $6.93(3.2-9.2)$ & $8.1-10.7 \mathrm{mmol} / \mathrm{l}$ & $7.17(4.6-10.7)$ & $74 \%(108)$ \\
\hline
\end{tabular}

\section{Imaging diagnostics}

A detailed list of imaging techniques comparing their success rates at detecting THA and TKA infections is contained in Fig. 2.

In a total of 119 conventional hip X-rays $(n=73)$, infection was suspected in $61 \%(n=73)$ while loosening of the implant was revealed in $55 \%(n=66)$. Knee X-rays were taken on 118 patients. Suspected infection was found in 29 $\%$ of cases $(n=34)$. Signs of loosening were apparent in 31 $\%(n=37)$ Fig. 3.

Significant differences emerged between THA and TKA in the detection of implant loosening (THA $55 \%$ (66), TKA $31 \%$ (37); p <0.001) and suspicion of infection by a radiologist (61\% (73), TKA $29 \%$ (34); p <0.001), with infection after THA more likely to be detected.

CT scans were produced of 18 hip joints, of which $94 \%$ (17) showed signs of infection. CT scans were performed on 5 knee joints, with positive results being achieved in 80 $\%$ (4). There were no significant differences between the two groups in terms of CT $(\mathrm{p}=0.52)$.

MRI was used for diagnosis even less frequently: in 6 cases on the hip joint and 1 case on a knee joint. All the MRI scans positively indicated suspected infection.

The most common type of nuclear medicine examination carried out was FDG-PET-CT. Of the 44 hip joints examined, infection was suspected in $97 \%$ (43). Infection was

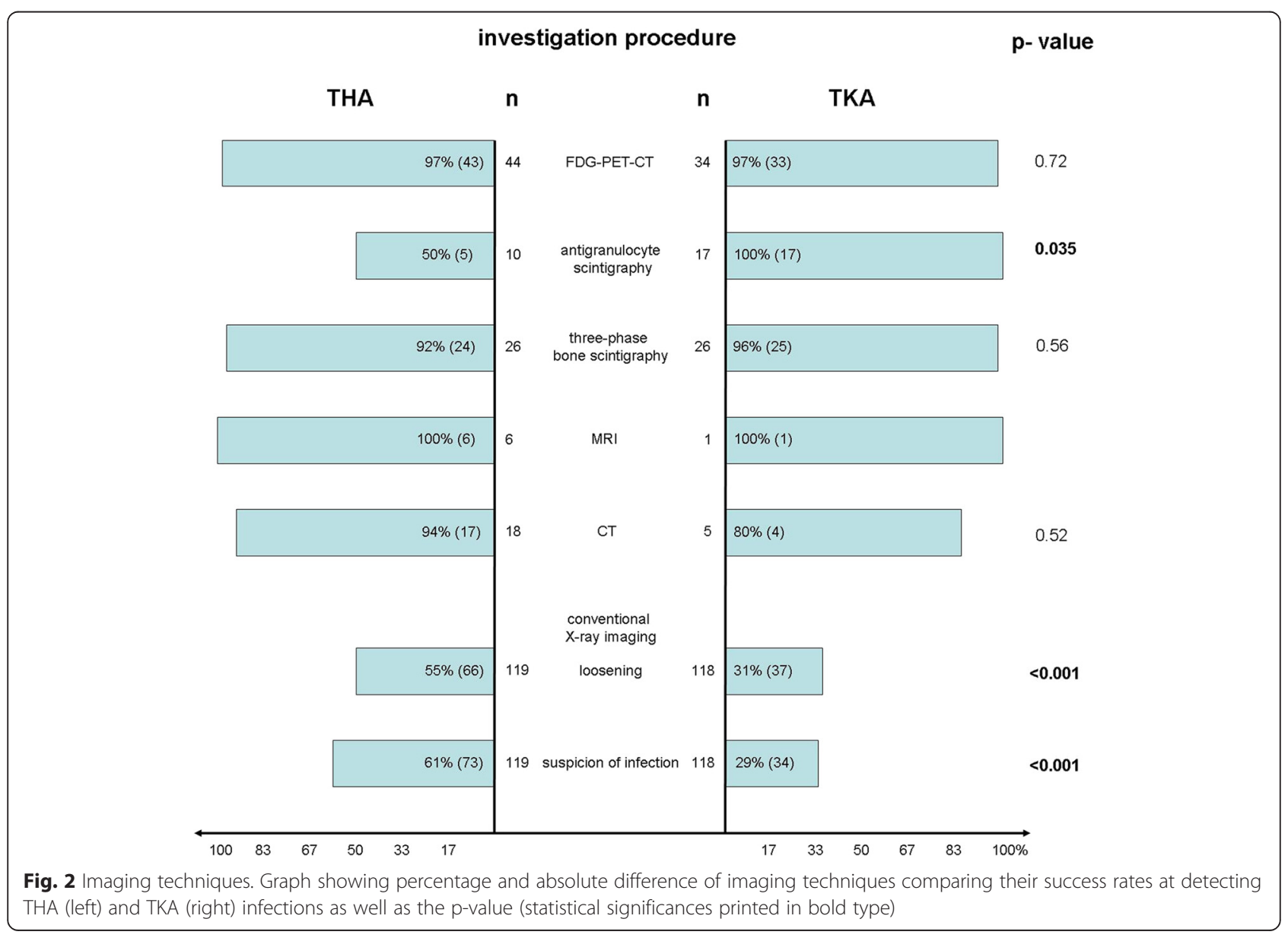




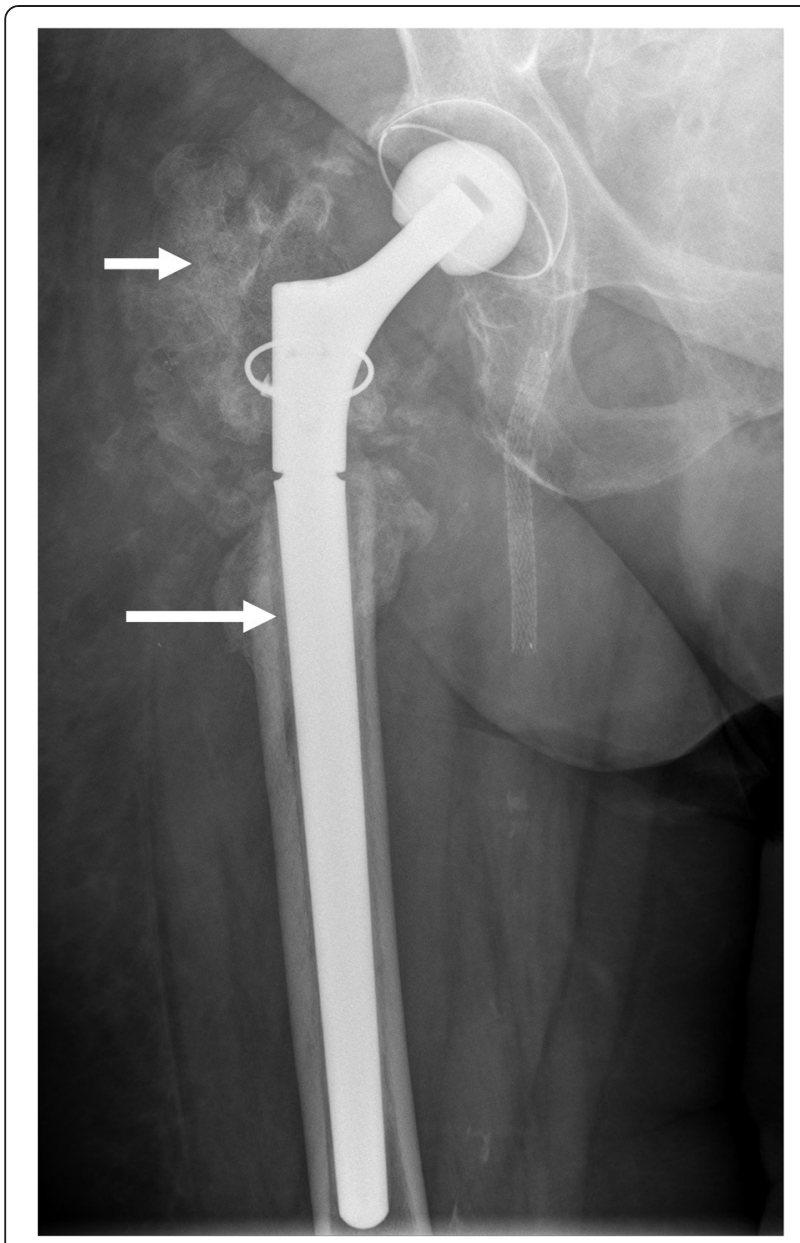

Fig. 3 Conventional hip X-rays. Showing a conventional hip X-rays with existing infection with loosening of the implant (long bolt) and periarticular ossifications (short bolt)

also indicated by 33 of the 34 knee examinations (97\%). There were no significant differences $(\mathrm{p}=0.72)$ Fig. 4.

Three-phase bone scintigraphy was performed on 26 hips and 26 knees. It was positive in $92 \%(\mathrm{n}=24)$ of hips and $96 \%(\mathrm{n}=25)$ of knees $(\mathrm{p}=0.56)$ Fig. 5.

Anti-granulocyte scintigraphy was carried out on 10 THA and 17 TKA patients. Significant differences were ascertained between THA and TKA regarding suspected infection according to a nuclear medicine specialist (THA $50 \%$ (5), TKA $100 \%$ (17); p = 0.035) in favour of TKA.

\section{Diagnostic arthrocentesis}

Arthrocentesis was performed on 85 patients with THA infection. The specimens were all analysed microbiologically. In addition, 53 specimens underwent histological evaluation.

Arthrocentesis was performed on 115 patients with TKA infection. The specimens were all analysed microbiologically while histological valuation was also carried out on 74 specimens. The positive and negative findings for THA and TKA infections are compared in Table 2.

Regarding the correlation between microbiology and histology, of the 63 positive microbiological cultures following THA infection, positive histology was observed in $60 \%$ (38 cases), too. However, in three cases (5\%), the histological findings were negative despite positive microbiological cultures. In 9 arthrocentesis specimens with no positive microbiological culture (41 \%), histological findings still indicated suspected infection.

Following TKA infection, positive histology also occurred in $58 \%$ (50 cases) of all positive microbiological cultures. By contrast, no histological signs of infection were found in $9 \%$ (8) of cases. Of the negative microbiological cultures, $45 \%$ (13 cases) nevertheless showed positive histology.

\section{Discussion}

Strikingly, our study found that significantly more patients with TKA infection suffered swelling, erythema and a burning sensation in the affected joint than THA patients Fig. 1, whereas, hardly any differences existed concerning pain or dysfunction. This is because the smaller layer of soft tissue on the knee joint leads to symptoms of localized inflammatory response showing earlier [7] while non-specific symptoms such as pain and dysfunction are experienced on the hip before any local inflammatory reaction becomes visible on the skin [15]. However, no differences emerged between systemic signs of infection such as general malaise, fever or sepsis, the systemic involvement of which is possible in connection with both THA and TKA infection. On the other hand, fistulas were found to occur significantly more often in connection with THA infection. This is probably because the infection is often silent and only noticed when the fistula opens. In the knee joint, a local inflammatory response is much more likely, prompting the patient to seek medical advice and treatment before a fistula can develop [16]. Furthermore, the clinical symptoms of PJI vary greatly, affecting the general clinical symptoms of inflammation [14]. Especially in chronic cases, they may be non-specific and are often misinterpreted in connection with previous pain in the joint [14]. In fact, up to $30 \%$ of prosthetic infections show no clinical symptoms whatsoever. Therefore, pain in the replaced joint accompanied by fever must always be properly diagnosed [9].

The first diagnostic tool used is mostly conventional $\mathrm{X}$-ray. Although X-rays can detect loosened implants, they can only sometimes distinguish between septic or aseptic loosening, making them ineffective for early infections [17]. Moreover, any existing periarticular ossifications only show up after a long time and do not prove an existing infection [18]. In our analysis, X-rays revealed significantly more loosenings and suspected 


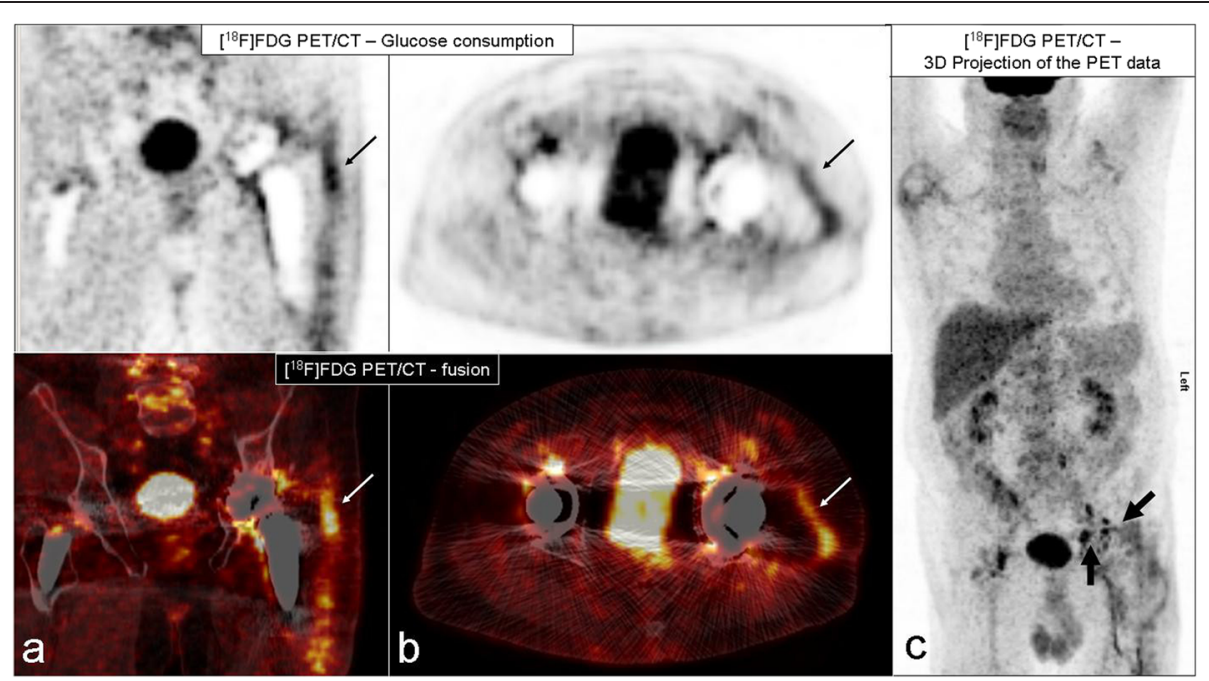

Fig. 4 PET/CT images. Representative [18 F] FDG PET/CT images of a patient with infection of the left total hip arthroplasty in coronal (a) and transversal (b) orientation (c) shows a 3D projection (maximum intensity projection) of the [18 F] FDG PET data. The images demonstrate a streaky intensive hypermetabolism in the soft parts surrounding the total hip arthroplasty (thin arrows) and focal hypermetabolisms in reactive inguinal lymph nodes (bold arrows)

infections on replacement hips than knees - partly because symptoms following THA take longer to develop [14,16] Fig. 3.

Although magnetic resonance imaging (MRI) is considered the technique of choice for soft tissue assessment in inflammatory events, interference from adjacent metallic implants impedes accurate assessment [19].
Substantial interference has even been reported in connection with non-ferromagnetic implants, such as titanium [20]. Therefore, MRI only seems suitable for specific problems such as abscesses in soft tissue [21]. CT also shows significant metal artefacts and therefore only plays a minor role in the diagnosis of PJI. It may be useful to assess any resulting changes to bone or to plan

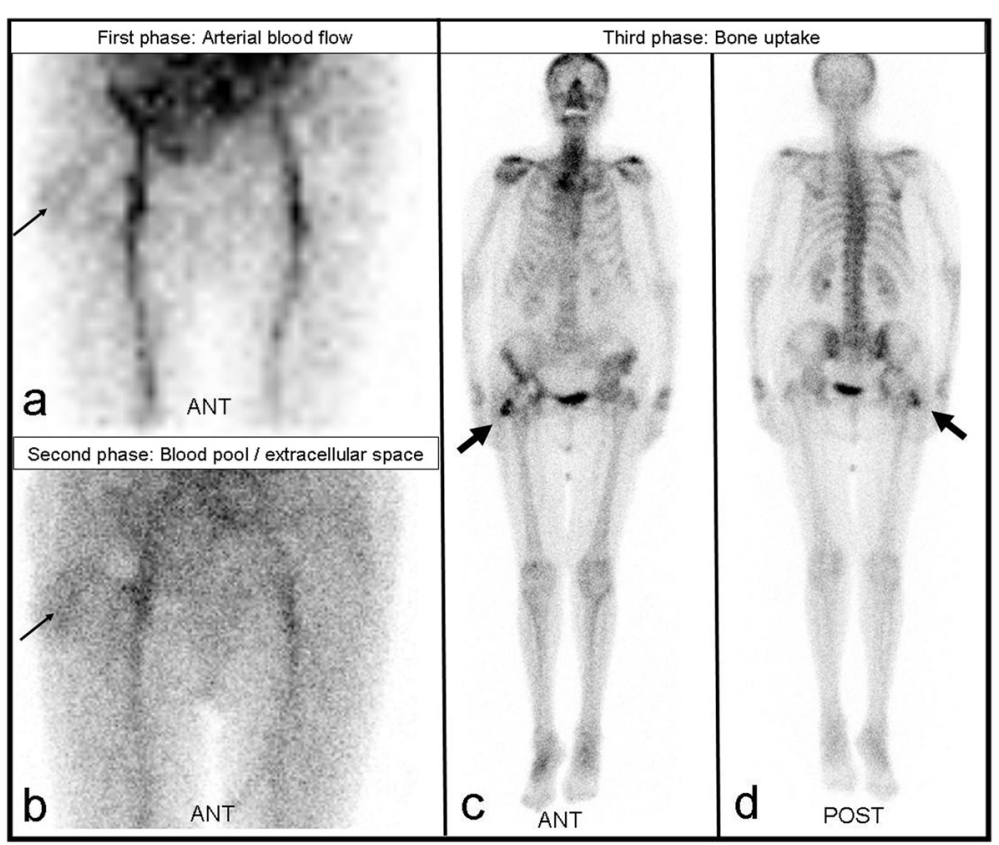

Fig. 5 Three-phase bone scintigraphy. [99mTc] DPD three-phase bone scintigraphy. Increased blood flow (a) and blood pool (b) in the soft tissue of the right hip, lateral of the total hip arthroplasty (thin arrows) as well as increased bone uptake (c, $\mathbf{d}$ ) in the lateral parts of the right hip (bold arrows) in a patient with infection of the right total hip arthroplasty 
Table 2 Microbiology and histology. Shows the positive and negative findings (percentage and absolute) for THA and TKA infections in microbiology and histology as well as the p-value

\begin{tabular}{|c|c|c|c|c|c|c|}
\hline & \multicolumn{2}{|l|}{ Microbiology } & \multirow[t]{2}{*}{$\mathrm{p}$-value } & & \multicolumn{2}{|l|}{ Histology } \\
\hline & Positive & Negative & & & Positive & Negative \\
\hline THA $(n=85)$ & $63(74.5 \%)$ & 22 (25.5\%) & 0.019 & THA $(n=53)$ & 47 (88.7 \%) & $6(11.3 \%)$ \\
\hline TKA $(n=115)$ & 86 (74.8 \%) & 29 (25.2 \%) & 0.025 & TKA $(n=74)$ & 63 (85.1\%) & 11 (14.9\%) \\
\hline
\end{tabular}

a possible explantation, but its use should be decided on a case-by-case basis [14,22].

Nuclear medicine techniques are still held in high regard and produce good or even very good results regarding specificity and sensitivity in the diagnosis of PJI, despite the varying nature of data in the literature [23]. The problem is that they are usually only useful at least six months postoperatively, making them unsuitable for diagnosing early infection [24].

Three-phase bone scintigraphy is a common and relatively inexpensive technique. It detects the enrichment

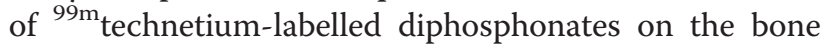
surface at three different times by means of a gamma camera. This enrichment depends on bone remodelling and is therefore more intensive if the endoprosthesis becomes loose. Accordingly, although this method is sensitive, it is also rather unspecific and provides no diagnostic evidence of a septic event, especially in the first twelve months after implantation [24,25]. Our study attributed showed good results to bone scintigraphy, the proportion of positively detected findings in THA and TKA exceeding $90 \%$. Then again, its accuracy is reported in the literature to be only $50-70 \%[24,25]$ Fig 5 .

In anti-granulocyte scintigraphy, ${ }^{99 \mathrm{~m}} \mathrm{Tc}$-labelled monoclonal anti-granulocyte antibodies are used, which detect the presence of granulocytes in inflamed tissue. This method is reported in the literature to have very high sensitivity and specificity of 95-100\% [26]. This could not be confirmed in our study, especially regarding THA infections, as positive results were not revealed in $50 \%$ of cases. The reason could not even be ascertained in a subsequent examination of the cases. There may be an anomaly involved which can partly be explained by the low number of cases of anti-granulocyte scintigraphy assessed $(n=10)$. By the way, two disadvantages of antigranulocyte scintigraphy are that it is expensive while its use of mouse antibodies sometimes triggers allergic reactions.

To improve specificity, scintigraphy can be combined with single-photon emission computed tomography (SPECT), a method which detects the distribution of a radioisotope in the body using planar imaging [26].

FDG-PET-CT showed especially good results in our study with $97 \%$ correctly positive results in both THA and TKA. In the literature, however, the accuracy of FDG-PET-CT is reported to vary greatly between 43 and
$92 \%$ [27,28]. Furthermore, its high costs and timeconsuming operation as well as the low proliferation of FDG-PET-CT scanners rule out its standard use in the diagnosis of PJI [29] Fig. 4.

Our study noted increased CRP levels in over $90 \%$ of cases of both THA and TKA infections. The mean value in both types of infections was about $100 \mathrm{mg} / \mathrm{l}(<5 \mathrm{mg} / \mathrm{l})$. Since CRP, being an acute phase protein, regularly increases non-specifically not just during infections but also after surgery, determining the CRP level is particularly suitable for monitoring development [30]. However, studies have found CRP to have a sensitivity of $91-93 \%$ but a sensitivity of just $83-86 \%$ in connection with infections [31].

Following arthroplasty, the increasing CRP reaches a maximum two or three days after surgery and returns to its normal range within three weeks [32]. A persistent rise or another increase are hence a sign of PJI and should always be properly diagnosed [19]. Further evidence of infection may be an increased white blood cell count (standard $4-10 \times 10^{6} / \mathrm{l}$ ), but this has low specificity [33]. However, fewer than half of all the patients in this survey showed an increase in leukocytes.

Procalcitonin and IL-6 are relatively new laboratory parameters with good specificity and sensitivity. But because only studies involving small numbers of cases have been performed so far, routine use is not recommended [34]. The advantage of procalcitonin is that it is a reliable marker of sepsis which allows the bacterial cause of sepsis to be diagnosed [34]. In our study, too, only about half the patients showed an increase in PCT. Although none of these laboratory parameters can reliably indicate or rule out PJI, they are important in order to monitor development [30]. Strikingly, over $70 \%$ of patients displayed falling $\mathrm{Hb}$ values, which can probably be attributed to infectious anaemia. This, too, is extremely nonspecific, but appeared to be more sensitive in our study than leukocytes and PCT.

Ultimately, only positive microbiological culture is regarded as a decisive criterion for diagnosis. The gold standard here is arthrocentesis [10]. Even so, the sensitivity and specificity of the results can vary greatly and depend on the type and quantity of extracted fluid and ongoing antibiotic therapy. Moreover, valid conclusions are often only possible after fourteen days' incubation, limiting its usage in acute diagnosis. The significantly 
higher positive values of histology compared to microbiology were noticeable regardless of whether the knee or the hip was involved (Table 2). This may be due to variations caused by antibiotic therapy beforehand or the insufficient amount of fluid extracted for microbiological cultivation [30]. Ultimately, our survey showed histological analysis to be clearly superior to microbiological evaluation. The main advantage of microbiological analysis is that it can quickly detect the pathogen present and its corresponding antimicrobial resistance so that drug-based therapy can start [30].

\section{Limitations}

The validity of this study is reduced by its retrospective design and the inhomogeneity of the patient population. Then again, this applies to the majority of patients with PJI. There was no exact differentiation of diagnosis depending on the time of infection, which may be particularly significant for imaging.

\section{Conclusion}

In particular, the clinical picture of PJI may be nonspecific and, especially on the hip, not be accompanied by typical symptoms of inflammation. In imaging, reliable diagnosis is often only possible after some time, making the morphological representation of early infections difficult. FDG-PT-CT proved especially useful and is likely to become even more important. There are currently no laboratory parameters which are suitable for the reliable primary diagnosis of PJI. However, the traditional inflammatory markers, especially CRP, are essential to monitor the course of infection. Arthrocentesis is obligatory to diagnose infection, and specimens should undergo both microbiological and histological examination.

\section{Consent}

All patients were informed and confirmed their approval.

\section{Competing interests}

The authors declare that they have no competing interests. We acknowledge support from the German Research Foundation (DFG) and Universität Leipzig within the program of Open Access Publishing.

\section{Authors' contributions}

DZ made substantial contributions to devising the study as well as the analysis and interpretation of the data. LW carried out the majority of data acquisition. TP, GVSS and MG were involved in drafting the manuscript. PB and ST were involved in creating the figures. AR, CJ and CEH gave final approval for the version to be published. The final manuscript has been read and approved by all the authors.

\section{Author details}

${ }^{1}$ Department of Orthopaedic Surgery, Traumatology and Plastic Surgery, University Hospital Leipzig, Liebigstrasse 20, 04103 Leipzig, Germany. ${ }^{2}$ Department of Nuclear medicine, University Hospital Leipzig, Liebigstrasse 20, 04103 Leipzig, Germany. ${ }^{3}$ Department of Diagnostic and Interventional Radiology, University Hospital Leipzig, Liebigstrasse 20, 04103 Leipzig, Germany.
Received: 5 February 2015 Accepted: 29 April 2015

Published online: 16 May 2015

\section{References}

1. Mota RE, Tarricone R, Ciani O, Bridges JF, Drummond M. Determinants of demand for total hip and knee arthroplasty: a systematic literature review. BMC Health Serv Res. 2012;12:225.

2. Tian W, DeJong G, Brown M, Hsieh CH, Zamfirov ZP, Horn SD. Looking upstream: factors shaping demand for postacute joint replacement rehabilitation. Arch Phys Med Rehab. 2009;90:1260-8.

3. Learmonth ID, Young C, Rorabeck $C$. The operation of the century: total hip replacement. Lancet. 2007;370(9597):1508-19.

4. Malchau H, Herberts P, Eisler T. The Swedish Total Hip Replacement Register. J Bone Joint Surg Am. 2002;84(2):2-20.

5. Kurtz SM, Lau E, Schmier J, Ong KL, Zhao K, Parvizi J. Infection burden for hip and knee arthroplasty in the United States. J Arthroplasty. 2008;23(7):984-91.

6. Garvin $\mathrm{KL}$, Hanssen $\mathrm{AD}$. Infection after total hip arthroplasty. Past, present, and future. J Bone Joint Surg Am. 1995;77(10):1576-88.

7. Maurer TB, Ochsner PE. Infected knee arthroplasty. A treatment algorithm at the Kantonsspital Liestal. Switzerland. Orthopade. 2006;35:917-28.

8. Zimmerli W, Trampuz A, Ochsner PE. Prosthetic-joint infections. N Engl J Med. 2004;351(16):1645-54.

9. Friesecke C, Wodtke J. Management of periprosthetic infection. Chirurg. 2008;79(8):777-92.

10. Militz M, Bühren V. Replacement of infected knee and hip endoprostheses. Chirurg. 2010;81(4):310-20.

11. Wodtke J, Löhr JF. The infected implant. Orthopade. 2008;37(3):257-67.

12. Barrack RL, Sawhney J, Hsu J, Cofield RH. Cost analysis of revision total hip arthroplasty. A 5-year followup study. Clin Orthop Relat Res. 1999;369:175-8.

13. Zimmerli W, Ochsner PE. Management of infection associated with prosthetic joints. Infection. 2003;31(2):99-108.

14. Laffer $R$, Ruef C. Diagnosis and treatment of prosthetic joint infections. Z Rheumatol. 2006;65(1):12. 14-17.

15. Itasaka T, Kawai A, Sato T, Mitani S, Inoue H. Diagnosis of infection after total hip arthroplasty. J Orthop Sci. 2001;6:320-6.

16. Gollwitzer H, Diehl P, Gerdesmeyer L, Mittelmeier W. Diagnostic strategies in cases of suspected periprosthetic infection of the knee. A review of the literature and current recommendations. Orthopade. 2006;35(9):904. 906-8, 910-6.

17. Love C, Marwin SE, Palestro CJ. Nuclear medicine and the infected joint replacement. Semin Nucl Med. 2009;39(1):66-78.

18. Ibrahim MS, Raja S, Khan MA, Haddad FS. A multidisciplinary team approach to two-stage revision for the infected hip replacement: a minimum five-year follow-up study. Bone Joint J. 2014;96(10):1312-8.

19. Trampuz A, Zimmerli W. Prosthetic joint infections: update in diagnosis and treatment. Swiss Med Wkly. 2005;135(17):243-51.

20. Del Pozo JL, Patel R. Clinical practice. Infection associated with prosthetic joints. N Engl J Med. 2009;361(8):787-94.

21. Hayter CL, Koff MF, Shah P, Koch KM, Miller TT, Potter HG. MRI after arthroplasty: comparison of MAVRIC and conventional fast spin-echo techniques. AJR Am J Roentgenol. 2011;197(3):405-11.

22. Cahir JG, Toms AP, Marshall TJ, Wimhurst J, Nolan J. CT and MRI of hip arthroplasty. Clin Radiol. 2007:62(12):1163-71.

23. Lima AL, Oliveira PR, Carvalho VC, Saconi ES, Cabrita HB, Rodrigues MB. Periprosthetic joint infections. Interdiscip Perspect Infect Dis. 2013;54:2796.

24. Love C, Tomas MB, Marwin SE, Pugliese PV, Palestro CJ. Role of nuclear medicine in diagnosis of the infected joint replacement. Radiographics. 2001;21(5):1229-38.

25. Palestro CJ, Torres MA. Radionuclide imaging in orthopedic infections. Semin Nucl Med. 1997;27:334-45.

26. Graute V, Feist M, Lehner S, Haug A, Müller PE, Bartenstein P, et al. Detection of low-grade prosthetic joint infections using 99mTc-antigranulocyte SPECT/CT: initial clinical results. Eur J Nucl Med Mol Imaging. 2010;37(9):1751-9.

27. Stumpe KD, Nötzli HP, Zanetti M, Kamel EM, Hany TF, Görres GW, et al. FDG PET for differentiation of infection and aseptic loosening in total hip replacements: comparison with conventional radiography and three-phase bone scintigraphy. Radiology. 2004;231(2):333-41.

28. Chacko TK, Zhuang H, Stevenson K, Moussavian B, Alavi A. The importance of the location of fluorodeoxyglucose uptake in periprosthetic infection in painful hip prostheses. Nucl Med Commun. 2002;23(9):851-5. 
29. Haenle M, Skripitz C, Mittelmeier W, Skripitz R. Economic impact of infected total hip arthroplasty in the German diagnosis-related groups system. Orthopade. 2012;41(6):467-76.

30. Frommelt L. Diagnosis and treatment of foreign-body-associated infection in orthopaedic surgery. Orthopade. 2009;38(9):806-11.

31. Greidanus NV, Masri BA, Garbuz DS, Wilson SD, McAlinden MG, Xu M, et al. Use of erythrocyte sedimentation rate and C-reactive protein level to diagnose infection before revision total knee arthroplasty. A prospective evaluation. J Bone Joint Surg Am. 2007:89(7):1409-16.

32. White J, Kelly M, Dunsmuir R. C-reactive protein level after total hip and total knee replacement. J Bone Joint Surg (Br). 1998;80(5):909-11.

33. Li SF, Cassidy C, Chang C, Gharib S, Torres J. Diagnostic utility of laboratory tests in septic arthritis. Emerg Med J. 2007;24(2):75-7.

34. Worthington T, Dunlop D, Casey A, Lambert R, Luscombe J, Elliott T. Serum procalcitonin, interleukin-6, soluble intercellular adhesin molecule-1 and lgG to short-chain exocellular lipoteichoic acid as predictors of infection in total joint prosthesis revision. Br J Biomed Sci. 2010;67(2):71-6.

\section{Submit your next manuscript to BioMed Central and take full advantage of:}

- Convenient online submission

- Thorough peer review

- No space constraints or color figure charges

- Immediate publication on acceptance

- Inclusion in PubMed, CAS, Scopus and Google Scholar

- Research which is freely available for redistribution 